\title{
High strain rate tensile testing of sheet materials using three Hopkinson pressure bars
}

\author{
M. Dunand ${ }^{1,2}$, G. Gary ${ }^{1}$, and D. Mohr ${ }^{1,2}$ \\ ${ }^{1}$ Solid Mechanics Laboratory (CNRS UMR 7649), Department of Mechanics, École Polytechnique, Palaiseau, \\ France \\ ${ }^{2}$ Impact and Crashworthiness Laboratory, Department of Mechanical Engineering, Massachusetts Institute of \\ Technology, Cambridge MA, USA
}

\begin{abstract}
In an attempt to circumvent the inherent problems associated with Split Hopkinson Tensile Bar (SHTB) systems, a new experimental technique is proposed for the high strain rate tensile testing of sheet specimens in Split Hopkinson Pressure Bar (SHPB) systems. Existing solutions employ a multiple gage section hat-shaped specimen to transform the incoming pulse from compression into tension at the specimen level. However, multiple gage section specimens are not suitable for studying the postnecking behavior of materials as the required symmetry of the mechanical system is no longer guaranteed in the post-necking range. Here, we present a new load-inversion device that is used in conjunction with three Hopkinson pressure bars for the tensile loading of single gage section sheet specimen. The device is designed to transform the compression load applied at its boundaries into a tensile loading of the specimen. Two output bars are used to measure the total force applied on the specimen boundaries, while Digital Image Correlation (DIC) is used to determine the strain histories on the specimen surface based on photographs acquired at a frequency of about $100 \mathrm{kHz}$. In addition to uniaxial tensile experiments on TRIP780 steel sheet specimens at strain rates ranging from 200/s to 1000/s, results are presented on the dynamic fracture testing of notched tensile specimens.
\end{abstract}

\section{Introduction}

The stress-strain response of engineering materials at high strain rates is typically determined based on uniaxial compression experiments on Split Hopkinson Pressure Bar (SHPB) systems [1]. In the case of sheet materials, it is difficult to perform a reliable dynamic compression experiment due to limitations associated with buckling. Different techniques have been developed in the past for the high strain rate tensile testing of materials (e.g. [2-8]). The key challenges lie in the generation of the tensile loading pulse and the attachment of the specimen to the Hopkinson bars.

This conference paper is an abridged version of reference [9]. We propose a new experimental technique for the tensile testing of a sheet specimen in a modified split Hopkinson Pressure Bar apparatus made of one input bar and two output bars. The proposed technique is particularly useful for the experimental characterization of the effect of high strain rates on the onset of fracture of ductile sheet materials, where large strains need to be reached. The specimen features only one gage section which allows for the measurement of the post-necking response at high strain rates.

\section{Experimental procedure}

\subsection{Load inversion device}

The load inversion device is designed to be positioned between the input and output bars of a SHPB system to perform dynamic tension tests. The sheet specimen is attached to the load inversion device, while there is no need for attachments between the device and the bars (transmission of compression through flat contact surfaces only). The proposed device is shown in Fig. 1. Its two main components are:

(1) A "pusher" (green) which is positioned between the specimen and the input bar. It comprises a cover plate (grey) and eight M4-12.9 screws which apply the clamping pressure necessary to hold the specimen. The pusher inverts the incoming compressive pulse into a tensile loading of the specimen. With the clamping plate attached, it features the same cross-sectional area as the $20 \mathrm{~mm}$ diameter input bar.

(1) A "stirrup" (red) which holds the specimen and transmits the applied force to the transmitter bars. Each leg of the stirrup matches the cross-sectional area of the corresponding output bar. The shape of the transverse section of the stirrup is optimized to minimize its inertia while limiting its bending during loading, in order to reduce perturbations on the measured force. Note that a slot of the thickness of the sheet specimen has been cut into the stirrup instead of using a floating clamp.

A base plate along with a set of linear guides is used as a positioning system to ensure accurate alignment of the pusher and the stirrup. The linear guides are also used to reduce the deflection of the pusher due to bending.

\subsection{Split Hopkinson pressure bar (SHPB) system}

The SHPB system consists of a $1.2 \mathrm{~m}$ long striker bar and a $3 \mathrm{~m}$ long input bar (20 mm dia.). Two $2 \mathrm{~m}$ long output bars (14 mm dia.) are used. The recorded strain signals are post-processed with the software package DAVID [10] to reconstruct the time histories of strain at the end of the incident and transmitter bars, following the procedures described in [11]. Accounting for wave dispersion, the 


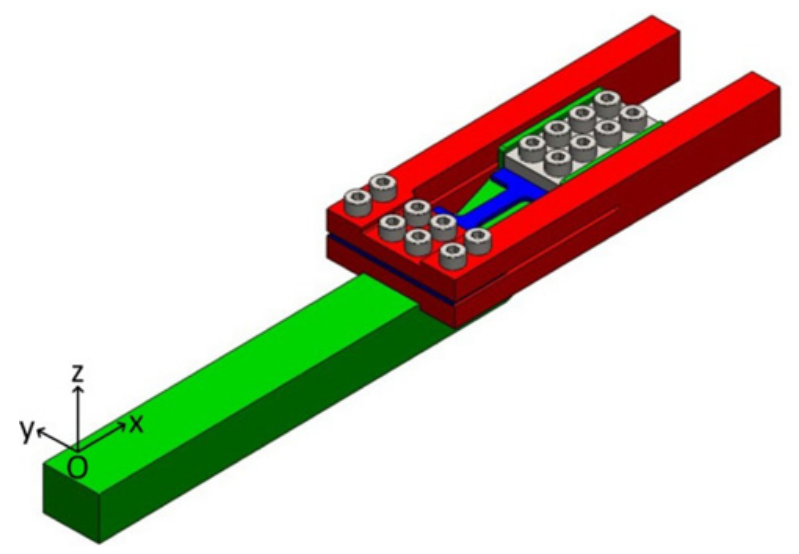

Fig. 1. Schematic of the Load Inverting Device (LID) including the pusher (green), the stirrup (red) and the specimen (blue).

measured incident and reflected waves are "transported" to the incident bar/loading device interface. Analogously, the measured transmitted waves in the output bars are transported to the output bar/loading device interfaces.

To measure displacements and strains within the specimen gage section, a high speed video system (Phantom v7.3 with $90 \mathrm{~mm}$ macro lenses) is employed. The virtual extensometer function of the digital image correlation software VIC2D (Correlated Solutions, SC) is used to determine the relative displacement of two points located in the axis of symmetry of the specimen gage section. The output force history is used to estimate the stress within the specimen. Note that the after the onset of necking (e.g. after reaching the maximum engineering stress), the measured engineering strain must be interpreted as normalized relative displacement. Inverse analysis is needed to determine the stress-strain curve in the post-necking range.

\section{Numerical validation}

A detailed finite element analysis of a dynamic experiment is performed to assess the validity of the experimental technique. As compared to the experimental validation, the FEA-based validation has the advantage of validating the experimental design independent of possible practical problems associated with the alignment of parts or the clamping of the specimens. Our 3D model of the experimental setup includes the specimen ( $1.4 \mathrm{~mm}$ thickness, $5 \mathrm{~mm}$ width, $15 \mathrm{~mm}$ gage length), the load inverting device as well as the input and output bars. The striker bar impact is not modeled. Instead, the measured incident wave pulse is directly applied to the free end of the input bar.

The explicit solver of the finite element analysis software Abaqus (2007) is used to run the analysis. All parts are meshed with reduced integration $3 \mathrm{D}$ solid elements (types C3D8R from the Abaqus element library). The mesh size is chosen such as to allow for an accurate description of axial frequencies of up to $50 \mathrm{kHz}$ and possible bending waves. A tie constraint is defined between the specimen and the LID to represent a slip-free rigid attachment. The specimen material (TRIP780 steel) is

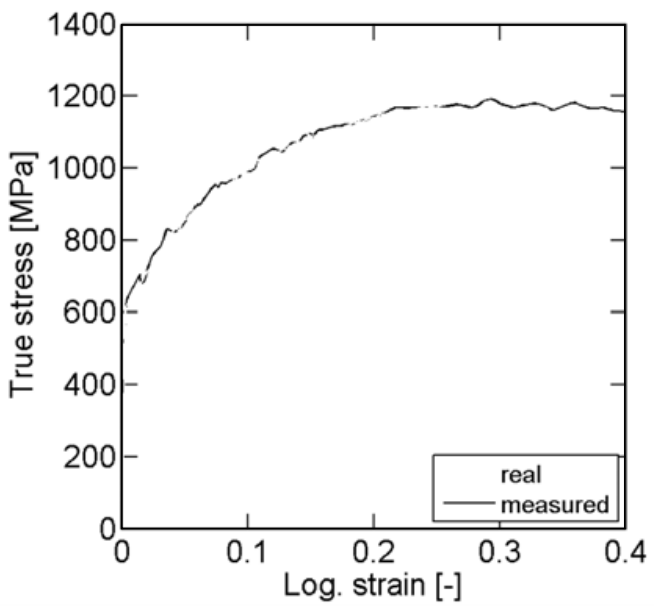

Fig. 2. FE-based validation of the experimental technique for uniaxial tension with an equivalent plastic strain rate of about 900/s: real and measured stress-strain curves.

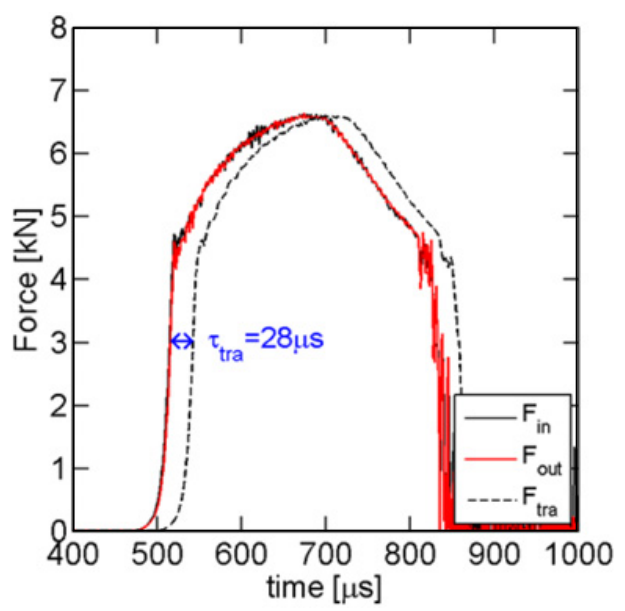

Fig. 3. Forces applied at the gage section boundaries by the pusher (solid black line), by the stirrup (solid red curve) and at the output bars/stirrup interface (dashed black line).

modeled as elasto-plastic assuming rate-independent J2plasticity. The loading pulse is applied at the free end of the input bar as surface pressure history. It corresponds to a stress pulse measured experimentally for a striker velocity of $14.8 \mathrm{~m} / \mathrm{s}$, corresponding to an equivalent plastic strain rate of about $860 / \mathrm{s}$ before necking.

Figure 3 compares the determined true stress versus logarithmic strain curve (solid black line) with the actual material behavior (solid red line). The two curves lie almost perfectly on top of each other up to a strain of about 0.25 (onset of necking), which confirms the validity of the proposed experimental technique for the determination of the stress-strain response of a typical AHSS sheet material at a strain rate of $900 / \mathrm{s}$. I

To evaluate the assumption of quasi-static equilibrium, we determined the time history of the axial forces applied at each boundary of the specimen gage section. The two solid curves in Fig. 4 lie almost perfectly on top of each other, which indicates that the hypothesis of quasi-static equilibrium of the specimen gage section during loading holds true. In addition, the force applied at 


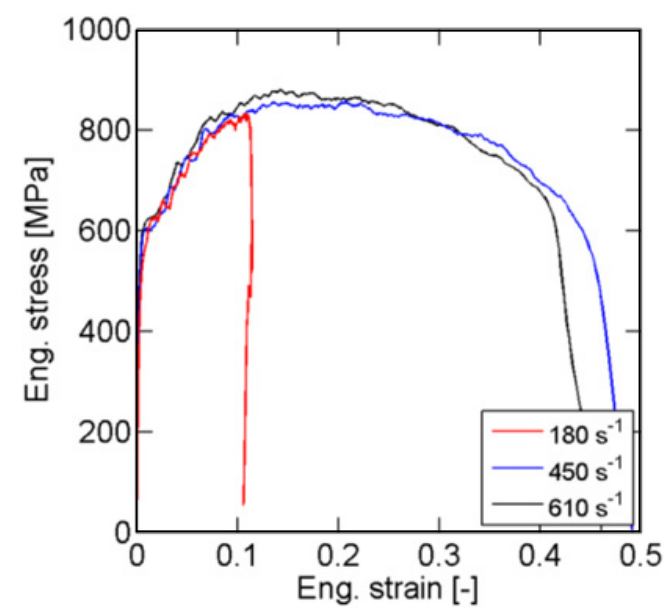

Fig. 4. Engineering stress-strain curves for different plastic strain rates.

the stirrup/transmitter bar interface is shown as a dashed line showing a delay of $\tau_{\text {tra }} \cong 28 \mu$ s which corresponds to the duration associated with the wave travel through the stirrup.

\section{Experimental validation}

All experiments are performed on a TRIP780 steel provided by POSCO (Korea) in the form of $1.4 \mathrm{~mm}$ thick sheets. Low strain rate experiments have been carried out on an electro-mechanical universal testing machine (MTS model G45) at a pre-necking strain rate of $5 \times 10-4 / \mathrm{s}$.

\subsection{High strain rate uniaxial tension experiments}

The uniaxial tension specimens ( $5 \mathrm{~mm}$ width, $15 \mathrm{~mm}$ gage length) are extracted from $1.4 \mathrm{~mm}$ thick TRIP780 steel sheets through water jet cutting. The specimen loading axis is aligned with the sheet rolling direction. The dynamic experiments are carried out with striker velocities of 4.2, 11.8 and $14.6 \mathrm{~m} / \mathrm{s}$. The experimental results are summarized in Fig. 5. The respective average pre-necking strain rates for the present experiments are 180/s, 450/s and 610/s. Except for the experiment at $185 / \mathrm{s}$, this duration of the loading pulse was long enough to deform the specimens all the way to failure. The onset of necking (characterized by the force maximum) is observed at an engineering strain of about 0.16 for all experiments. The three curves lie approximately on top of each other, indicating that the material exhibits no noticeable effect of strain rate on the flow stress and strain hardening for the range of high strain rates considered (from 180/s to 610/s). Observe that the experimentally-measured stress-time signals exhibit only small oscillations.

\subsection{Notched tension experiments}

For illustration, dynamic experiments are performed on notched tensile specimens $(10 \mathrm{~mm} \mathrm{~min}$. gage width, $6.67 \mathrm{~mm}$ notch radius). These experiments allow for the characterization of the effect of stress state on ductile

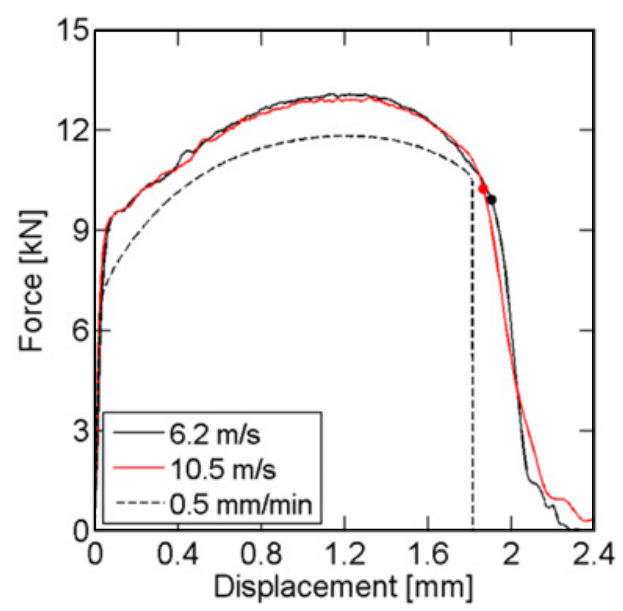

Fig. 5. Experiments on a notched tensile specimen: Forcedisplacement curves for high strain rate loading at different striker velocity (solid lines) and for a low strain rate experiment (dashed line).

fracture (see [12] for details). Experiments are performed with striker velocities of $6.2 \mathrm{~m} / \mathrm{s}$ and $10.5 \mathrm{~m} / \mathrm{s}$. Throughout each experiment, the relative axial displacement between two points on the specimen shoulders is measured using DIC and synchronized with the corresponding axial force history measurements. The obtained force-displacement curves for high strain rate loading are shown in Fig. 6 (solid lines) next to the curve for low strain rate loading (dashed line, crosshead velocity of $0.5 \mathrm{~mm} / \mathrm{min}$ ). As for the uniaxial tension experiments, the results of the two high strain rate experiments are almost identical. However, the force level is about $10 \%$ higher than that measured in the low strain rate experiment. The solid dots indicate the instant of onset of fracture as determined through high speed photography.

\section{Conclusions}

An experimental technique is proposed to determine the stress-strain curve for sheet materials at high strain rates. The experiments are performed on a conventional split Hopkinson pressure bar system with the help of a newdesigned load inversion device. The device comprises a pusher which is in contact with the input bar and a stirrup which is in contact with two output bars. The specimen is clamped between the pusher and the stirrup. Two output bars are used to minimize oscillations in the output force measurements due to bending. The strains in the specimen gage section are determined from the digital correlation of high speed video images. The experimental technique is validated both numerically and experimentally for strain rates of up to $1000 / \mathrm{s}$. In addition, the experimental technique is employed to perform a dynamic fracture experiment on a notched tensile specimen.

\section{Acknowledgements}

The partial financial support of the French National Center for Scientific Research (CNRS) and the MIT/Industry fracture consortium is gratefully acknowledged. Professors Tomasz 
Wierzbicki (MIT) and Bengt Lundberg (Uppsala University) are thanked for valuable discussion. Mr. Philippe Chevalier from Ecole Polytechnique is thanked for his assistance in carrying out the experimental work.

\section{References}

1. H. Kolsky, Proceedings of the Physical Society. Section B 62, 676 (1949)

2. J. Harding, E.O. Wood, J. Campbell, J. Mech. Eng. Science 2, 88 (1960)
3. T. Nicholas, Exp. Mech. 21, 177 (1981)

4. U. Lindholm, L. Yeakley, Exp. Mech. 8, 1 (1968)

5. D. Mohr, G. Gary, Exp. Mech. 47, 681 (2007)

6. P. Mouro, G. Gary, H. Zhao, J. Phys. IV France 10 (2000)

7. G. Staab, A. Gilat, Exp. Mech. 31, 232 (1991)

8. K. Ogawa, Exp. Mech 24, 81 (1984)

9. M. Dunand, G. Gary, D. Mohr, submitted (2012)

10. G. Gary, David User's Manual (2005)

11. H. Zhao, G. Gary, Mat. Sci. Eng. A 207, 46 (1996)

12. M. Dunand, D. Mohr, Int. J. Solids Struct. 47, 1130 (2010) 